\title{
Late post-operative paraparesis after rib penetration of the spinal canal in a patient with neurofibromatous scoliosis
}

\author{
M. Cappella $\cdot$ N. Bettini $\cdot$ E. Dema $\cdot$ \\ M. Girardo $\cdot$ S. Cervellati
}

Received: 12 August 2007/Accepted: 17 March 2008/Published online: 10 May 2008

(C) Springer-Verlag 2008

\begin{abstract}
Rib displacement into the spinal canal is a rare cause of paraplegia or paraparesis in patients affected by neurofibromatous scoliosis. We describe a case of paraparesis in a 14-year-old child affected by neurofibromatous dystrophic kyphoscoliosis, treated with combined posterior and anterior spinal arthrodesis. Seventeen days after the surgical treatment the patient developed clinical signs and symptoms of paraparesis. A CT scan showed the head of the fifth rib protruding into the spinal canal with cord compression. Rib resection and posterior cord decompression were carried out following complete neurological recovery.
\end{abstract}

Keywords Kyphosis - Neurofibromatosis - Paraparesis · Rib displacement $\cdot$ Scoliosis

\section{Introduction}

Spinal deformity is the most common musculoskeletal manifestation in neurofibromatosis type I or peripheral neurofibromatosis with the incidence ranging from 10 to $60 \%$ [1]. Spinal deformities in patients affected by neurofibromatosis have been divided into dystrophic and nondystrophic forms [2]. Nondystrophic deformities behave similar to idiopathic scoliosis, but a close follow-up is firmly required because dystrophic changes may develop with time. This ability of a spinal deformity to transform by acquiring dystrophic features is called "modulation", that has been reported to occur in $81 \%$ of children who present

M. Cappella $(\bowtie) \cdot$ N. Bettini · E. Dema $\cdot$ M. Girardo · S. Cervellati

Department of Spine Surgery, Centro di Chirurgia Vertebrale, Hesperia Hospital, Via Arquà 80/A, 41100 Modena, Italy e-mail: camax@katamail.com spinal deformity before age seven and $25 \%$ of those who are diagnosed after age seven [3]. Dystrophic features are characterized by a rapid and relentless progression and conservative treatment is usually ineffective $[2,3]$. Common features of dystrophic deformities are represented by short thoracic curve with severe apical rotation, severe kyphosis, vertebral scalloping, pencilling of the ribs, foraminal enlargement, widening of the spinal canal, spindled transverse processes, defective pedicles, and presence of paravertebral tumors [1]. Spinal cord compression with paraplegia or paraparesis is uncommon in patients affected by dystrophic deformities and it was described as caused by severe kyphosis with vertebral subluxation, neurofibroma protruding through the foramina and ribs penetration into the spinal canal [1].

In this paper, we report a rare case of spastic paraparesis due to spinal cord compression caused by rib penetration in the spinal canal of a child with dystrophic deformity that was treated by posterior and anterior spinal arthrodesis.

\section{Case report}

The patient is a 14-year-old boy affected by type I neurofibromatosis and kyphoscoliosis. He was treated with Milwaukee brace since the age of ten. At the age of 13 he began to suffer from weakness of the lower limbs and exhibited progressive difficulty on walking about. The radiological investigation confirmed the presence of a double thoracic kyphoscoliosis. The thoracic kyphosis measured $120^{\circ}$ with the apex at the level of T5-T6 (Fig. 1a, b). The magnetic resonance imaging showed a noticeable segmentary cord compression at the level of T5-T6 and excluded the presence of intraspinal tumours (Fig. 2a, b). 
Fig. 1 A 14-year-old child with neurofibromatous

kyphoscoliosis and worsening paraparesis. The A-P (a) and lateral (b) radiographs show the severe spinal deformiy
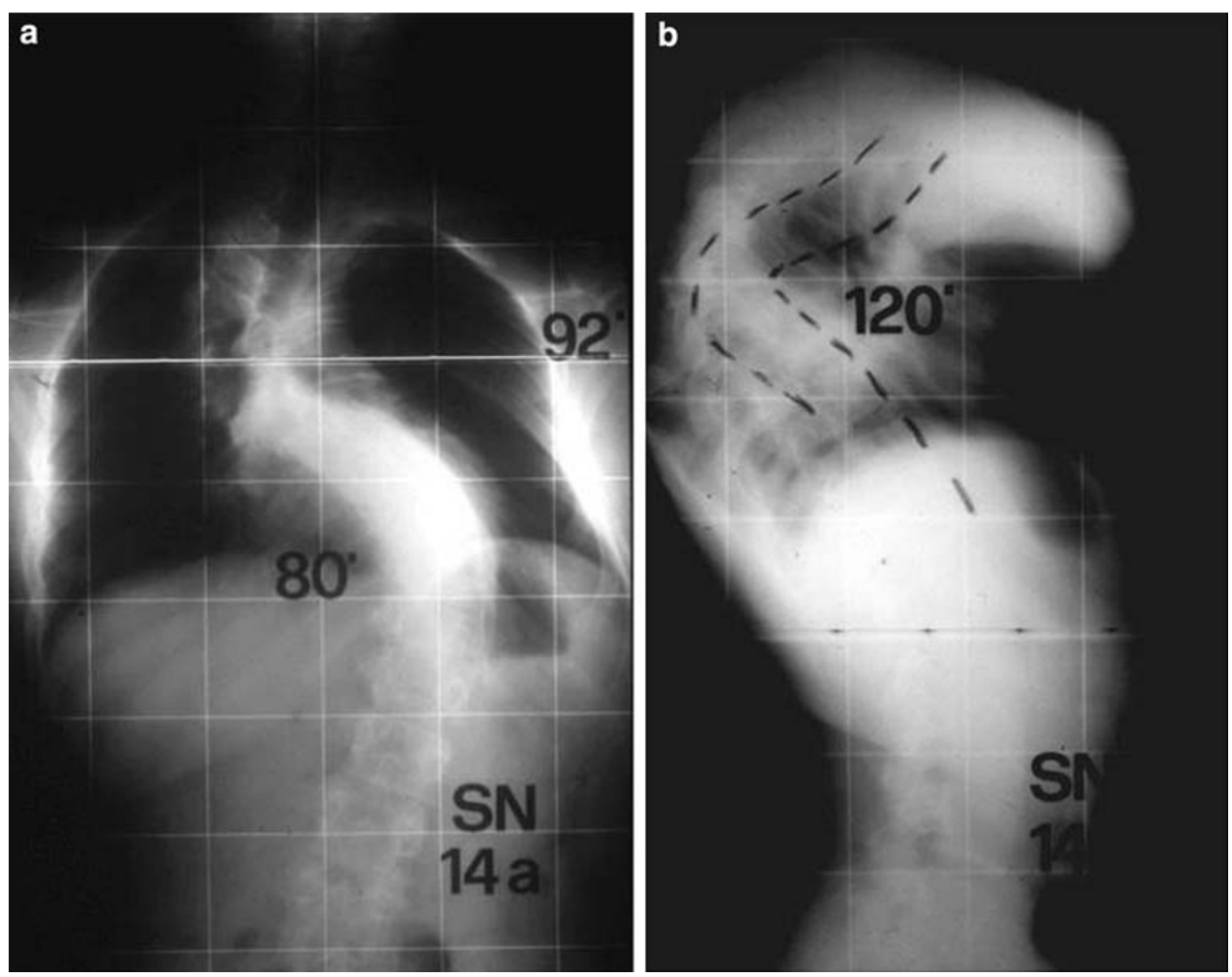

We performed surgical correction and instrumented posterior arthrodesis (T1-L1) using abundant bone graft. The postoperative period was regular without any neurological complication and patient was released from the hospital on the ninth postoperative day. A body cast was applied to contain any movement for a period of 2 months, during which he had complete neurological recovery. Fifty days after posterior surgery, we performed anterior arthrodesis by an open thoracotomy. The patient was released from hospital on seventh day with a body cast. Ten days later the patient complained of lower limbs weakness and he rapidly exhibited paraparesis. He was then admitted in the emergency room and X-rays showed the right position of spinal instrumentation. The CT scan confirmed that instrumentation as well as the anterior rib bone graft was perfectly inserted. Nevertheless, we observed a significant narrowing of the vertebral canal and a vertebral-rib malformation at the apex of the kyphosis (Fig. 3a). The three-dimension CT scan evidenced the head of the fifth rib penetrating the vertebral canal and cord compression (Fig. 3b). Resection of the rib, fragment removal and posterior cord decompression were carried out. Three days after we observed a noticeable improvement of neurological state and a complete neurological recovery then followed. The patient worn body casts and a Milwaukee brace till 9 months after surgery. At last control, 2 years after surgery, the patient was able to walk without any limitation.

\section{Discussion}

The most common pattern of neurofibromatous dystrophic deformities is represented by a single short thoracic curve and abnormal kyphosis or lordosis [2], characterized by rapid progression, that usually develops despite the appropriate conservative treatment. Neurological deficits may be a consequence of severe kyphosis with vertebral subluxation, cord compression by neurofibroma protruding through the foramina and ribs penetration into the spinal canal [1].

Rib displacement into the spinal canal is an uncommon cause of cord compression and, to our knowledge, only few cases have been previously described with a variable involvement of the neurological functions [4-12]. The higher rate of rib displacement seems to be observed at the apex of the curves, where maximal rotation of vertebral bodies is present $[10,12]$.

The present case report appears to be noteworthy because a complete neurological recovery was firstly obtained by posterior instrumented arthrodesis, regardless of the rib displacement into the spinal canal, that was not evidenced by preoperative radiological evaluation. This led us to consider the severe kyphosis as the cause of neurological deficit. Actually, after CT scan assessment revealed intraforaminal rib displacement causing cord compression, a careful re-evaluation of pre-operative MRI permitted us to evidence this complication (Fig. 2b). 
Fig. 2 a Preoperative MRI evidenced cord compression at the level of T5-T6 and excluded the presence of intraspinal tumours. b A careful examination of MRI permits to identify the rib displacement into the spinal canal (white arrow)
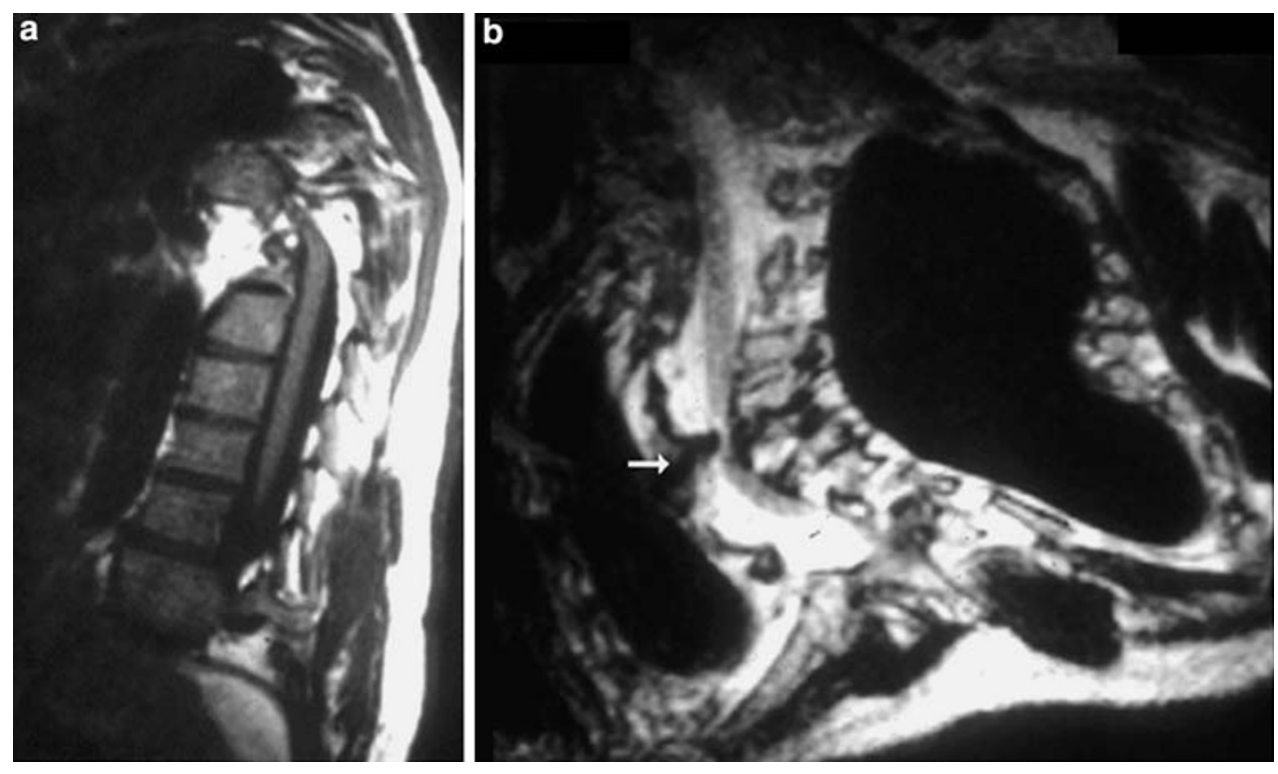

Fig. 3 a The CT scan showed the fifth rib penetration into the enlarged intervertebral foramina (black arrow). b The 3D CT scan confirmed the rib displacement into the spinal canal (white arrow)
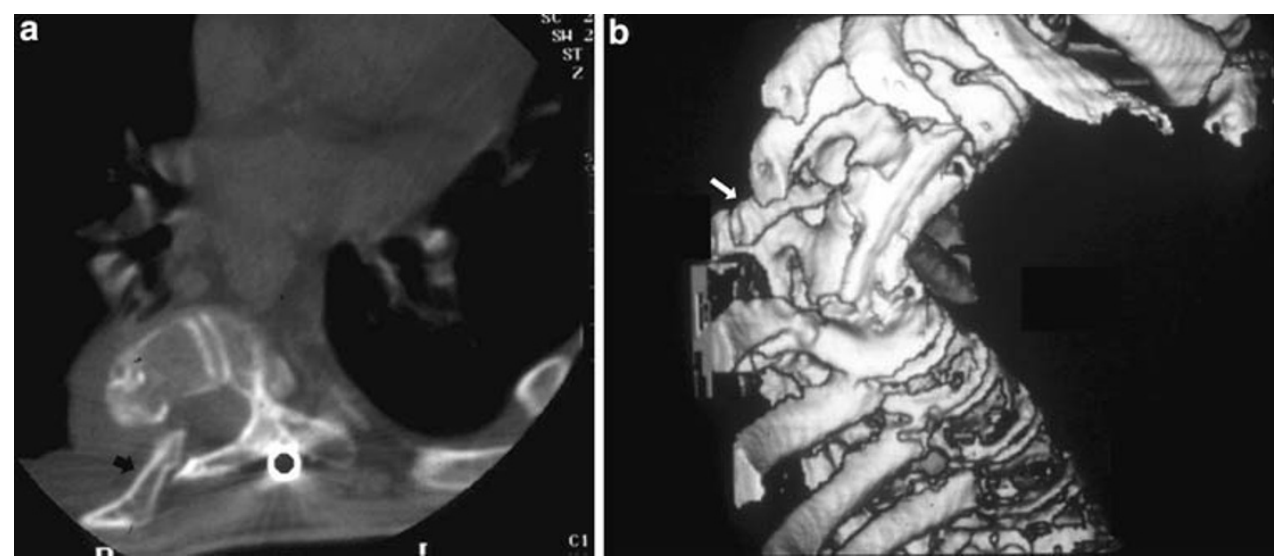

Furthermore, the period that followed the posterior instrumented arthodesis did not raise any suspect about the abnormal position of the fifth rib because of the absence of neurological signs or symptoms. Finally, lower limbs paraparesis appeared 17 days after anterior fusion without any promoting factor.

As evidenced by all previous reported cases, rib displacement into the spinal canal may have different patterns of clinical presentation [4-12]. It may be of acute or insidious onset $[5,7]$ and surgical treatment does not seem to be related with the occurrence of cord compression.

Although rib displacement into the spinal canal represents an infrequent complication of neurofibromatous spinal deformities, it must be excluded during preoperative radiological evaluation, even in the absence of neurological deficits. In fact, the presence of a rib displacement would require the surgical resection of the rib in addition to the spinal arthrodesis. To this purpose, a pre-operative study with the use of radiographs and MRI scan is recommended in order to identify the abnormality. Even if MRI does not seem to be specific, it may raise concerns of the lesion, by a careful observation of axial and sagittal images of thoracic spine, with a particular attention in the examination at the apex of the curve. At last, a guided CT scan permits to confirm the presence of rib displacement into the spinal canal.

Conflict of interest statement The authors declare that they have no conflict of interest related to the publication of this manuscript.

\section{References}

1. Kim HW, Weinstein SL (1997) The management of scoliosis in neurofibromatosis. Spine 22(23):2770-2776

2. Funasaki H, Winter RB, Lonstein JB, Denis F (1994) Pathophysiology of spinal deformities in nuerofibromatosis. $\mathrm{J}$ Bone Joint Surg (Am) 76:692-700

3. Durrani AA, Crawford AH, Chouhdry SN, Saifuddin A, Morley TR (2000) Modulation of spinal deformities in patients with neurofibromatosis type 1 . Spine 25:69-75

4. Flood BM, Butt WP, Dickson RA (1986) Rib penetration of the intervertebral foraminae in neurofibromatosis. Spine 11:172-174 
5. Major MR, Huizenga BA (1988) Spinal cord compression by displaced ribs in neurofibromatosis. J Bone Joint Surg (Am) 70:1100-1102

6. Uno K, Sha T, Shiraishi H, Shiba R, Kumon H (1994) Space occupying lesion in the spinal canal associated with spinal deformities in neurofibromatosis. J Jpn Scoliosis Soc 158-162

7. Deguchi M, Kawakami N, Saito H, Arao K, Mimatsu K, Iwata H (1995) Paraparesis after rib penetration of the spinal canal in neurofibromatous scoliosis. J Spinal Disord 8(5):363367

8. Dacher JN, Zakine S, Monroc M, Eurin D, Lechevallier J, Le Dosseur P (1995) Rib displacement treatening the spianal cord in a scoliotic child with neurofibromatosis. Pediatr Radiol 25:58-59
9. Kamath SV, Kleinman PK, Ragland RL, Tenreiro -Picon OR, Knor JR (1995) Intraspinal disclocation of the rib in neurofibromatosis: a case report. Pediatr Radiol 25:538-539

10. Khoshhal KI, Ellis RD (2000) Paraparesis after posterior spinal fusion in neurofibromatosis secondary to rib displacement: case report and literature review. J Pediatr Orthop 20:799-801

11. Legrand B, Filipe G, Blamoutier A, Khouri N, Mary P (2003) Intraspinal rib penetration in four patients in neurofibromatosis vertebral deformities. Rev Chir Orthop Reparatrice Appar Mot 89(1):57-61

12. Mukhtar IA, Letts M, Kontio K (2005) Spinal cord impingement by a displaced rib in scoliosis due to neurofibromatosis. Can J Surg 48(5):414-415 